\title{
EARLY ASSESSMENT OF VIIRS ON-ORBIT CALIBRATION AND SUPPORT ACTIVITIES
}

\author{
Xiaoxiong Xiong ${ }^{1}$, Kwofu Chiang ${ }^{2}$, Jeffrey McIntire ${ }^{2}$, Hassan Oudrari ${ }^{2}$, Aisheng $\mathrm{Wu}^{2}$, \\ Mathew Schwaller ${ }^{1}$, and James Butler ${ }^{1}$ \\ ${ }^{1}$ Sciences and Exploration Directorate, NASA/GSFC, Greenbelt, MD 20771, USA \\ ${ }^{2}$ Sigma Space Corporation, 4600 Forbes Boulevard, Lanham, MD 20706, USA
}

\begin{abstract}
The Suomi National Polar-orbiting Partnership (S-NPP) satellite, formally the National Polar-orbiting Operational Environmental Satellite System (NPOESS) Preparatory Project (NPP), provides a bridge between current and future low-Earth orbiting weather and environmental observation satellite systems. The NASA's NPP VIIRS Characterization Support Team (VCST) is designed to assess the long term geometric and radiometric performance of the Visible Infrared Imaging Radiometer Suite (VIIRS) instrument onboard the S-NPP spacecraft and to support NPP Science Team Principal Investigators (PI) for their independent evaluation of VIIRS Environmental Data Records (EDRs). This paper provides an overview of Suomi NPP VIIRS onorbit calibration activities and examples of sensor initial onorbit performance. It focuses on the radiometric calibration support activities and capabilities provided by the NASA VCST.
\end{abstract}

\section{INTRODUCTION}

The Suomi National Polar-orbiting Partnership (S-NPP) spacecraft was launched on October 28, 2011 from the Vandenberg Air Force Base (VAFB) in California. The Visible Infrared Imaging Radiometer Suite (VIIRS) is one of the five sensors onboard the NPP spacecraft. It makes observations in 22 spectral bands with wavelengths ranging from 0.41 to $12 \mu \mathrm{m}$ and generates 22 Environmental Data Records (EDRs) from its calibrated and geolocated reflectance and radiance, also referred to as the Sensor Data Records (SDRs). VIIRS data products will enable a broad range of applications, including environmental and hazard monitoring and numerical weather prediction (NWP). Following a series of spacecraft activation and functional testing activities, VIIRS was turned on successfully on November 8, 2011 with its nadir door opened on November 21, 2011. During the first few months, the VIIRS has been going through a series of Intensive Calibration and Validation (ICV) activities.

The NASA NPP Instrument Calibration Support Element (NICSE) was initially established within the NPP
Science Data Segment (SDS) to independently evaluate VIIRS on-orbit radiometric and geometric performance and validate its Sensor Data Records (SDR), and to support the NPP science (Land, Ocean, and Atmosphere) Product Evaluation and Analysis Tools Elements (PEATE) for their on-orbit data product calibration and validation efforts [1-3]. As part of the NASA's NPP VIIRS calibration efforts, the roles and responsibilities of NICSE have been expanded and integrated into the NASA VIIRS Characterization Support Team (VCST). Following the transition from the NPOESS program to the Joint Polar Satellite System (JPSS), the VCST has been tasked to support a government-led VIIRS SDR calibration and validation (Cal/Val) team. Figure 1 depicts the overall VCST system architecture and interface with NASA SDS, NOAA, NPP Science Team, VIIRS $\mathrm{Cal} / \mathrm{Val}$, and NPP Mission Operation Team (MOT).

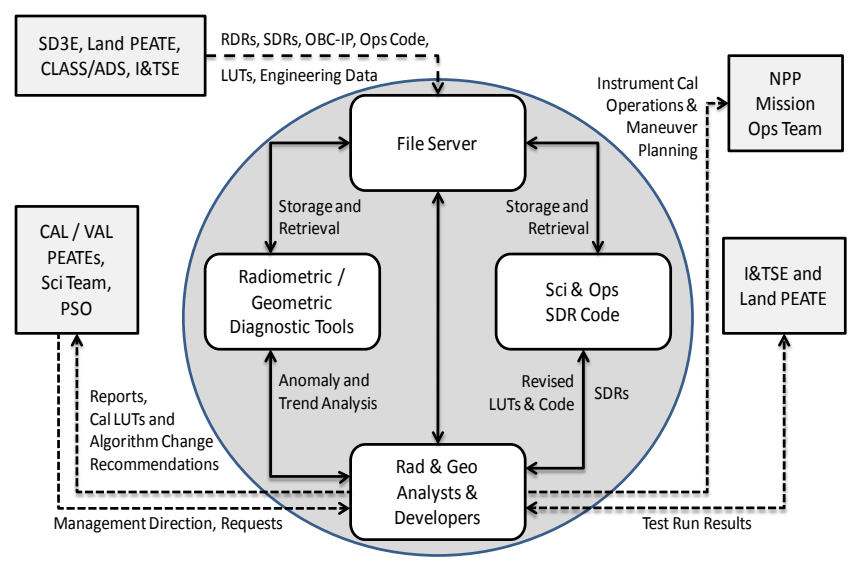

Fig. 1 VCST and Suomi-NPP NASA Science Data Segment (SDS) System Architecture and Interface for VIIRS Calibration Support.

\section{ON-ORBIT CALIBRATION ACTIVITIES}

VIIRS carries a set of on-board calibrators (OBC) with strong design heritage to the NASA's EOS Moderate Resolution Imaging Spectroradiometer (MODIS) currently operated on both Terra and Aqua spacecraft. VIIRS OBC include a solar diffuser (SD) and a solar diffuser stability 
monitor (SDSM) for the reflective solar bands (RSB) calibration and a blackbody (BB) for the thermal emissive bands (TEB) calibration [4]. Plus, a space view (SV) port provides measurements of sensor's background and signal offsets. The VIIRS front optical subsystem is a Rotating Telescope Assembly (RTA), followed by a half angle mirror (HAM), which rotates at half speed of the RTA. The VIIRS design allows the SD calibration data to be collected every orbit. The SDSM can also be operated every orbit or at an as needed frequency. It is designed to track on-orbit changes in the SD bi-directional reflectance factor (BRF). The on-board $\mathrm{BB}$, used for the TEB scan-to-scan radiometric calibration, is nominally controlled at a fixed temperature of $292.5 \mathrm{~K}$. Like MODIS, its warm-up and cool-down (WUCD) operations, during which the $\mathrm{BB}$ temperatures varying from instrument ambient to $315 \mathrm{~K}$, are conducted periodically.

During its intensive calibration and validation (ICV) period, the NPP spacecraft has planned and implemented three different types of maneuvers in support of the VIIRS on-orbit calibration and validation effort, (1) a series of yaw maneuvers to validate SD BRF, SD attenuation screen (SAS) transmission, and SDSM screen transmission; (2) a pitch maneuver to validate the TEB response versus scanangle (RVS); and (3) roll maneuvers for lunar observations to independently monitor the RSB calibration stability. In order to support the implementation of calibration maneuvers, a special VIIRS maneuver planner tool has been developed based on a similar tool originally designed and used for MODIS operation and calibration [5].

Also performed during VIIRS ICV period are a number of special tests designed to help resolve issues identified from sensor early on-orbit operations. The VCST has provided independent and quick data analyses to support VIIRS sensor characterization and calibration. Because of its experience and lessons learned from MODIS calibration and characterization, the VCST has quickly identified a number of important issues and discrepancies in the SDR operational production codes, reported the discrepancy and provided solutions for the fix through the discrepancy report assessment team (DRAT) at the JPSS Data Products and Algorithms (DPA) group.

\section{EARLY ON-ORBIT PERFORMANCE}

During NPP post-launch ICV period, the VCST has worked very closely with the government-led VIIRS SDR Cal/Val team in providing coordinated launch readiness support and independent validation of VIIRS on-orbit performance. The SD and SDSM calibrations were operated every orbit during the ICV period. Starting from the very beginning, the NASA calibration team has been independently analyzing the SD/SDSM data sets and regularly producing calibration trending results and diagnostic charts for the project and SDR community. Prior to opening the cryogenic cooler door, the ICV has focused on the RSB calibration. Figure 2 shows examples of VIIRS RSB radiometric calibration coefficients derived from SD on-orbit observations. Figure 2 (a) is the SD coefficient (F-factor) trending of imaging band 2 (I2) centered at $0.865 \mu \mathrm{m}$ and Figure 2 (b) is the middle detector gain factor (or 1/F) trending of all VisNIR bands for the High Gain state and the HAM side A.

Early in December 2011, the Aerospace Corp. $\mathrm{Cal} / \mathrm{Val}$ team reported an abnormal degradation greater than expected was seen in VisNIR detector responses. A number of special tests were performed immediately in December and January timeframe to help identify and characterize the root cause of the degradation. The discontinuities in the plots are resulted from these special tests. It was later discovered that the large degradation was due to flaws in the coating process used for the RTA mirrors. The amount of degradation is related to the accumulated solar exposure on to the RTA mirrors. After careful examination of VIIRS activity log to exclude the time period when RTA was not operated during the special tests or when instrument was in safe mode, Figure 2 (b) is re-plotted in 2 (c) to demonstrate the relationship of the degradation with effective solar exposure time. Because of this, the SDR calibration coefficients Look-Up-Table (LUT) are updated frequently by the $\mathrm{Cal} / \mathrm{Val}$ team to correct the degradation of RSB detector responses.

The SD coefficient F-factor is derived from SD calibration with correction applied to remove the impact due to solar diffuser degradation, which is monitored regularly by the SDSM. Figure 3 shows the solar diffuser degradation, or H-factor, trending derived from SDSM observations. The results are normalized to the first observation data point and to SDSM detector 8 centered at $0.926 \mu \mathrm{m}$. The degradation at this wavelength is expected to be extremely small, especially at mission beginning. The SDSM screen transmission parameters needed for to analyze and track SD degradation were measured pre-launch at limited viewing geometries and then characterized on-orbit using data collected during a series of yaw maneuvers made in 15 consecutive orbits on February 15 and 16 2012. Figure 4 illustrates SDSM detector 1 signals from the Sun view versus SDSM screen azimuth and elevation angles from all 15 yaws. The yaw maneuvers were successfully planned and executed and the data from these yaws have provided useful information and led to a more accurate SDSM screen transmission table used for $\mathrm{H}$-factor analysis and trending.

For TEB detectors, an on-board calibrator blackbody (OBC-BB) is used for scan to scan calibration. The Vgrooved $\mathrm{OBC}-\mathrm{BB}$ is a full aperture design similar to that of MODIS. The BB temperature is measured by 6 embedded thermistors and controlled at $292.5 \mathrm{~K}$ for nominal operation. Occasionally the BB will perform a WUCD operation, allowing its temperatures to be varied from instrument ambient ( 267 K) to $315 \mathrm{~K}$. Data collected from BB WUCD are used to validate and update if necessary the TEB nonlinear calibration coefficients. To date, two BB WUCD operations have been performed. Figure 5 shows the $\mathrm{BB}$ temperature profile during the WUCD activity in May 2012. 


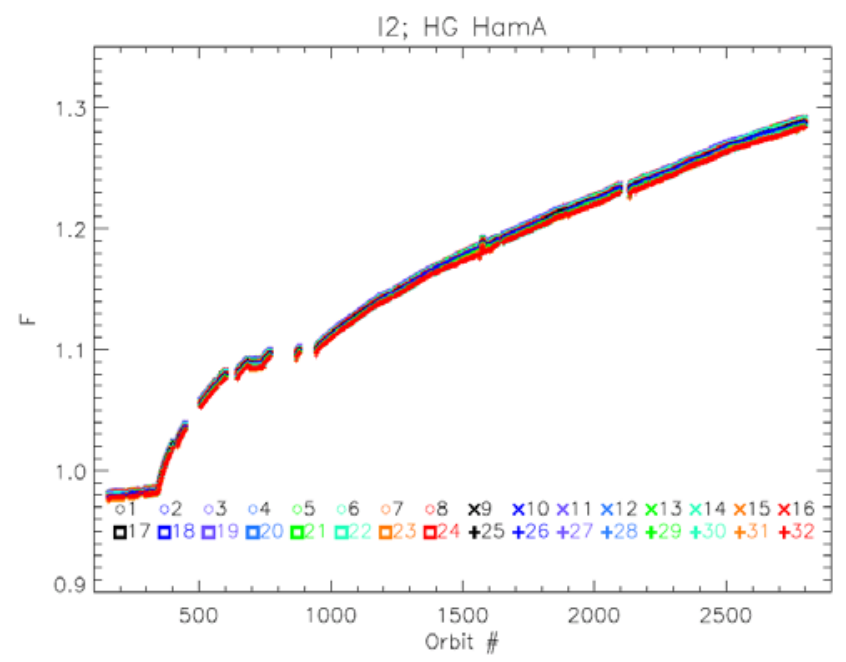

VIIRS VISNIR 1/F-factor; HG HamA
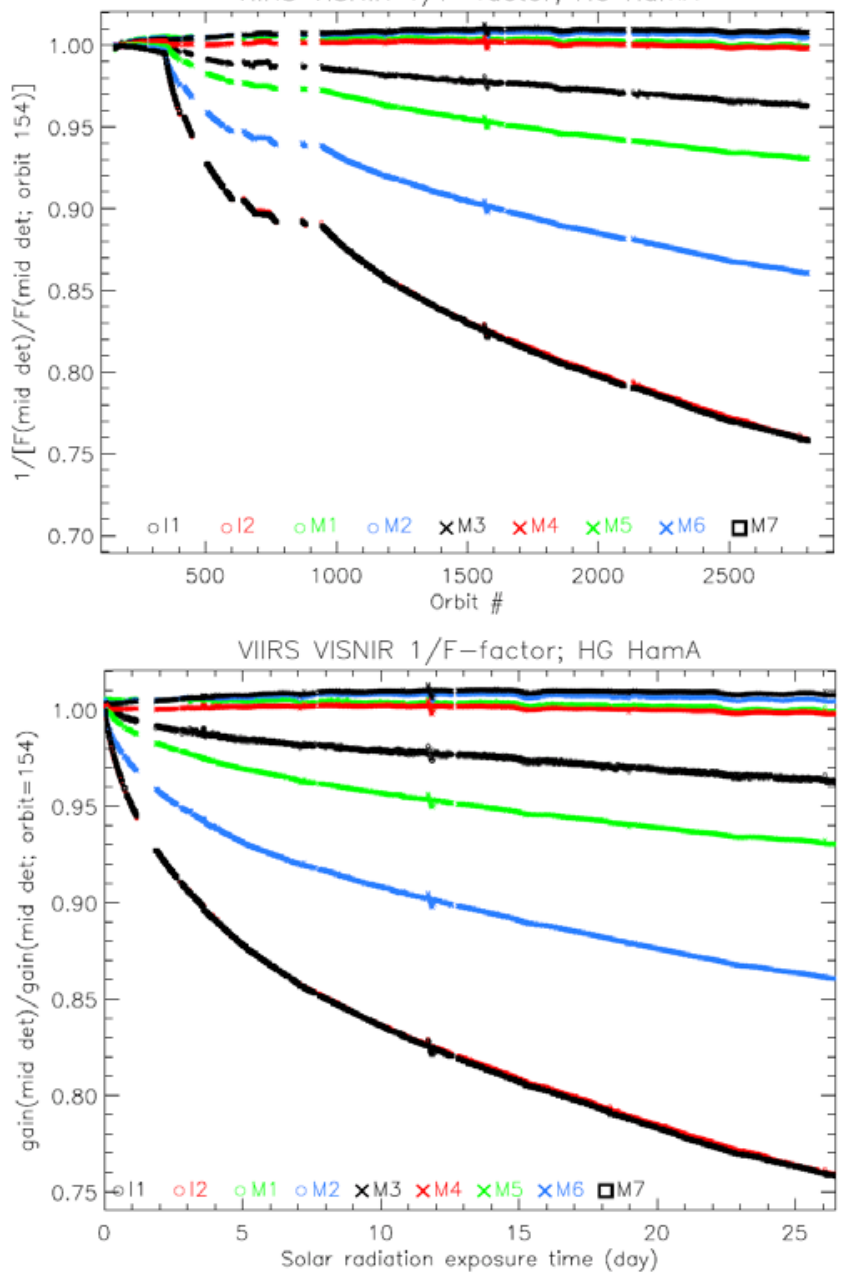

Fig. 2 (a) top: VIIRS I2 F-factor trending (all detectors, HAM side A); (b) middle: VisNIR bands (I1-I2, M1-M7) gain (1/F) trending (middle detector, high gain state, HAM side A); (c) bottom: gain (1/F) versus effective solar exposure time in days.

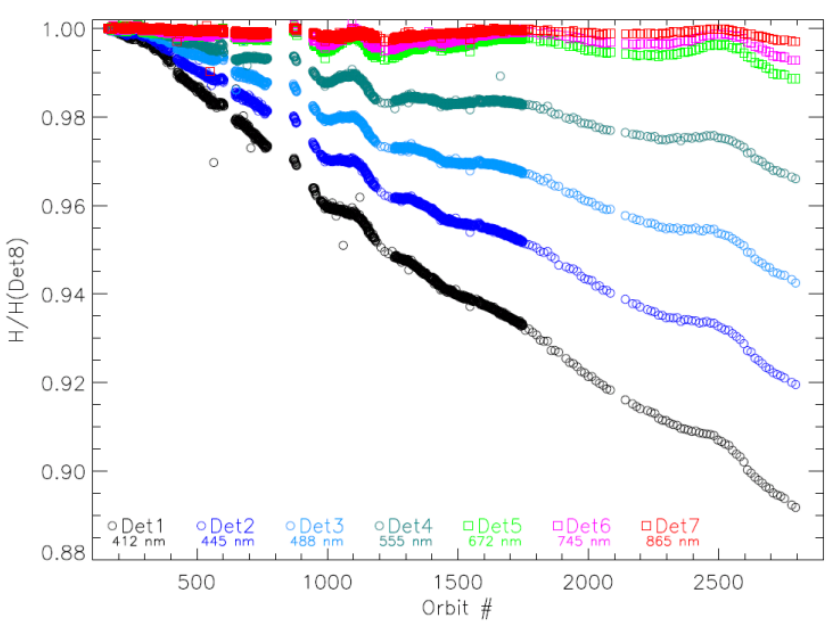

Fig. 3 Solar Diffuser degradation trending, normalized to SDSM Det 8 and to the first data point, by using updated SDSM screen transmission table derived from on-orbit yaw maneuvers.
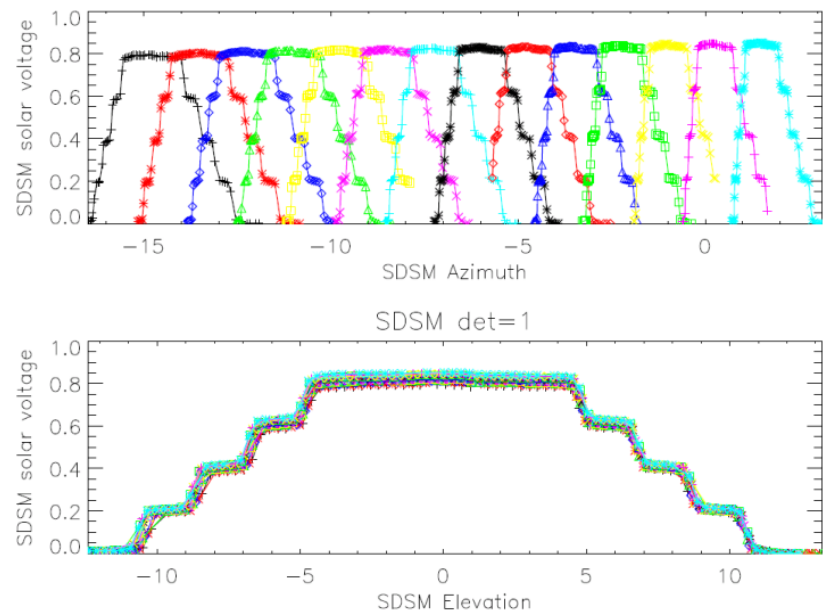

Fig. 4 SDSM detector response during yaw maneuvers. (a) top: Sun view signal vs. Azimuth angle of 15 oribits (yaw) range; (b) bottom: Sun view signal vs. Elevation angle.

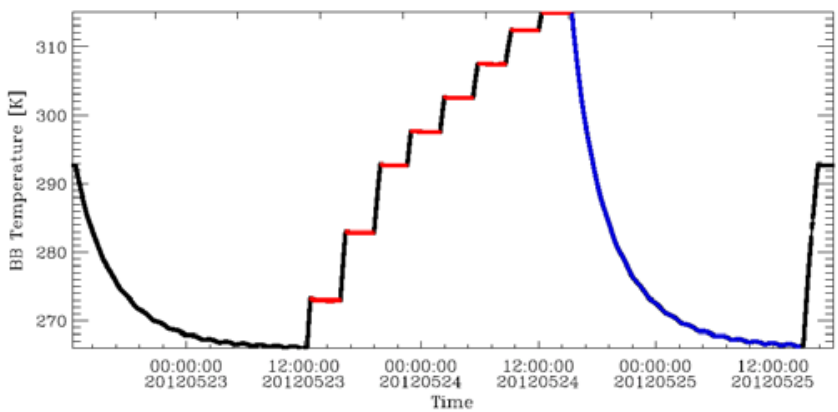

Fig. 5 OBC-BB warm-up cool-down temperature trending. During the warm-up period, the BB stays at 8 tmpeature plateaus for noise characterization and uniformity assessment. 
In addition to sensor response characterization, important telemetry and instrument temperatures that could impact sensor calibration or demonstrate instrument health are constantly monitored. Figure 6 plots the temperature trending of VisNIR focal plane assembly (FPA) for the first 6 months of on-orbit operation. All other telemetry temperatures used in SDR calibration codes, including BB, HAM, cavity, electronics, and focal planes are also monitored on a regular basis.

As described earlier, one of the calibration maneuvers is the lunar roll maneuver designed to view the Moon at a near monthly basis in support of RSB relative calibration. Thus far, the lunar roll maneuver planner tool developed by VCST has performed lunar predications and provided key maneuver parameters to the NPP MOT. The first regularly scheduled NPP VIIRS lunar calibration maneuver was executed on January 4, 2012 and the resulting lunar images of imaging band I1 are displayed in Figure 7. Since launch, 5 scheduled lunar calibration events have been successfully implemented. Sensor response trending derived from lunar observations has been in good agreement with that derived from SD calibration.

Other key radiometric calibration performance parameters, such detector signal to noise ratios (SNR) or noise equivalent difference temperatures (NEdT), detector response nonlinearity, and dynamic range have also been fully examined and characterized, all meeting the sensor design requirements despite of the degradation anomaly identified at mission beginning. In general, VIIRS on-orbit performance has been satisfactory and SDR data product quality continues to improve because of combined $\mathrm{Cal} / \mathrm{Val}$ efforts made by various groups.

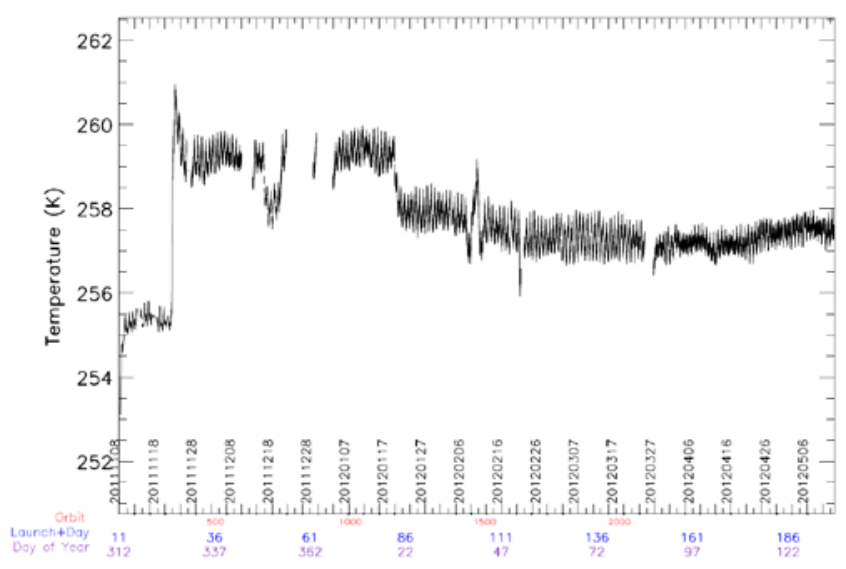

Fig. 6 Telemetry temperature trending of VisNIR warm focal plane assembly. The VisNIR FPA tenperature fluctuates with orbit and daily variation depending on ambient environment. Each data point represents granule average (48 scans).

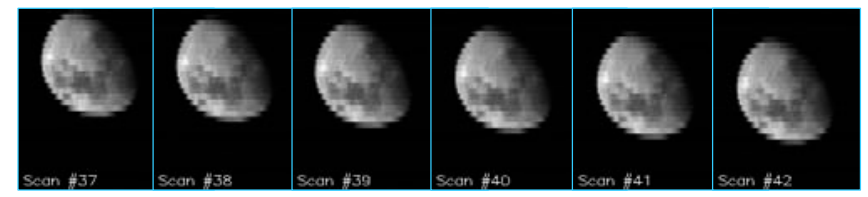

Fig. 7 Examples of NPP VIIRS lunar observations on Jan 4, 2012 for imaging band I1 (375m nadir resolution); 6 consecutive scans Moon images are displayed, 3 from HAM-A and 3 from HAM-B. Each image is consisted of 32 horizontal lines for 32 detectors.

\section{SUMMARY}

Overall VIIRS early on-orbit performance has been thoroughly assessed using data collected from its on-board calibrators, regularly scheduled lunar observations, and other calibration maneuvers. Through early on-orbit calibration and characterization activities, the NASA calibration team, including VCST, has clearly demonstrated its capability of providing independent evaluation of the VIIRS calibration and SDR product quality, and has met its design requirements of making recommendations for SDR operational code improvements and calibration coefficients LUT updates. VCST will continue to enhance its calibration and characterization capability, improve radiometric uncertainty quantification, refine the data analysis and trending tools, and explore better methodologies for SDR improvement over the lifetime of Suomi NPP mission.

\section{REFERENCES}

[1] X. Xiong, K. Chiang, J. McIntire, M. Schwaller, J. Butler, "Post-Launch Calibration Support For VIIRS Onboard NASA NPP Spacecraft," Geoscience and Remote Sensing Symposium, IGARSS, 2011.

[2] R. Sikorski, K. Chiang, M. Nishihama, R. Wolfe, X. Xiong, and M. Schwaller, "An Overview of NASA NPP SDS-NICSE Activities on VIIRS SDR Assessment,” Geoscience and Remote Sensing Symposium, IGARSS, 2010.

[3] K. Chiang, A. Wu, J. Sun, M. Schwaller, and X. Xiong, "Calibration Support for NPP SDR Assessment," Proceedings of the SPIE, Volume 7807, pp. 78071A-78071A-10, 2010.

[4] X. Xiong, K. Chiang, J. Esposito, B. Guenther, and W. Barnes, "MODIS On-orbit Calibration and Characterization," Metrologia 40, 89-92, 2003.

[5] Sun, J., X. Xiong, W. Barnes, and B. Guenther, MODIS Reflective Solar Bands On-Orbit Lunar Calibration, IEEE Trans. Geosci. Remote Sens., 45(7), 2383-2393, 2007. 